\title{
Generalized magnification in visual optics. Part 1: Magnification as linear transformation
}

\section{WF Harris}

Department of Optometry, University of Johannesburg, PO Box 524, Auckland Park, 2006 South Africa

$<$ wharris@uj.ac.za>

Submitted 24 March 2010; revised version accepted 31 August 2010

\begin{abstract}
In Gaussian optics magnification is a scalar; the interpretation is obvious. In linear optics, the simplest optics of astigmatic systems, the generalization is a $2 \times 2$ real matrix and, in general, is much harder to interpret. This generalized magnification may imply magnification in the familiar sense that differs from one meridian to another, shear distortion, rotation, reflection, inversion, magnification in the familiar sense or combinations of these effects.
\end{abstract}

The purpose of this paper is to illustrate generalized magnification and to provide a comprehensive interpretation. Because the treatment is abstract it can be applied to blur and size magnification and to any magnification that can be represented by a $2 \times 2$ matrix. (S Afr Optom 2010 69(3) 109-122)

Key words: Generalized magnification, astigmatism, rotation, reflection, inversion, linear magnification

\section{Introduction}

In Gaussian optics the student meets lateral (or transverse) magnification defined by ${ }^{1-6}$

$m=\frac{h^{\prime}}{h}$.

$h^{\prime}$ is the height of the image and h the height of the object. Transverse magnification is one of many types of magnification defined in Gaussian optics as a ratio of two scalars ${ }^{7}$. Scalars are sufficient for characterising the dimensions of object and image and other parameters. In linear optics, however, parameters are two dimensional; instead of scalars one has vectors. Because division by a vector is not defined definitions like that represented Equation 1 are not possible in linear optics.

Simple modification of Equation 1 to $m h=h^{\prime}$

does provide a definition suitable for generalization.
In linear optics the generalization takes the form ${ }^{8-15}$

$\mathbf{X x}=\mathbf{y}$.

Scalars $h$ and $h^{\prime}$ become vectors $\mathbf{x}$ and $\mathbf{y}$, perhaps representing the locations of corresponding points on an object and its image respectively; and scalar $m$ becomes a $2 \times 2$ matrix operator $\mathbf{X}$ that operates on $\mathbf{x}$ to produce $\mathbf{y}$. Thus $\mathbf{X}$ 'magnifies' $\mathbf{x}$ to $\mathbf{y}$. However, while magnification in Gaussian optics is easy to interpret generalized magnification in linear optics is not. What, for example, does a magnification of $\left(\begin{array}{cc}1 & 3 \\ 1 & -1\end{array}\right)$ mean? To some extent the term 'magnification' in this context is misleading. It may mean magnification in its everyday sense. But, in general it does not. It means any distortion, rotation, reflection, inversion or any combination of these effects which can be represented by the linear equation Equation 3 . 
We use it in the absence of any better word.

The purpose of this paper is to provide insight into the nature of this generalized magnification. Generalized magnifications of different kinds are illustrated and a method is presented for factorizing more complicated types into types that are conceptually simpler. The intention is to provide a comprehensive analysis of generalized magnification for future applications in a variety of ways in astigmatic optical systems.

The ideas are developed in terms of the effect the magnification has in magnifying object to image. However, because the approach here is abstract the conclusions are in fact applicable, with suitable modifications, to any process that can be represented by an equation of the form of Equation 3 including size magnification ${ }^{8-14}$, blur magnification ${ }^{11-14}$ and magnification of ray position and inclination across a system and cross magnifications of ray parameters ${ }^{15}$.

We begin with a numerical example; it will be referred to repeatedly in what follows.

\section{A numerical example}

Consider the magnification $\mathbf{X}=\left(\begin{array}{cc}1 & 3 \\ 1 & -1\end{array}\right)$. Let us examine its effect on several vectors $\mathbf{x}_{i}$. For $\mathbf{x}_{1}=\left(\begin{array}{l}3 \\ 0\end{array}\right)$

Equation 3 results in

$\mathbf{y}_{1}=\left(\begin{array}{cc}1 & 3 \\ 1 & -1\end{array}\right)\left(\begin{array}{l}3 \\ 0\end{array}\right)=\left(\begin{array}{l}3 \\ 3\end{array}\right)$.

$\mathbf{X}$ magnifies $\mathbf{x}_{1}$ to $\mathbf{y}_{1}$. Both vectors are plotted in Figure 1. The top and bottom entries of a vector are rectangular coordinates conveniently referred to as the horizontal and vertical components respectively. Similarly $\mathbf{X}$ magnifies $\mathbf{x}_{2}$ to $\mathbf{y}_{2}$, and so on for vectors $\mathbf{x}_{3}, \mathbf{x}_{4}$ and $\mathbf{x}_{5}$, all of the vectors being shown in Figure 1.

In most cases magnification by $\mathbf{X}$ causes stretching and rotation of the vector. Thus $\mathbf{x}_{1}$ is stretched and rotated anticlockwise to $\mathbf{y}_{1} \cdot \mathbf{x}_{2}$ and $\mathbf{x}_{4}$ are special, however, in that they are not rotated; magnification doubles the length of $\mathbf{x}_{2}$ and doubles the length and reverses $\mathbf{x}_{4} \cdot \mathbf{x}_{2}$ and $\mathbf{x}_{4}$ are, in fact examples of what are known in mathematics as the eigenvectors of matrix
$\mathbf{X}$; the factors 2 and -2 are corresponding eigenvalues ${ }^{16-18}$. $\mathbf{X}$ has two eigenvalues. Magnification by $\mathbf{X}$ magnifies by factor 2 in the direction of $\mathbf{x}_{2}$ and by factor 2 in the direction opposite that of $\mathbf{x}_{4}$.

Vectors $\mathbf{x}_{i}$ may represent points on an object, $\mathbf{y}_{i}$ and the locations of the corresponding points on the image that is the result of generalized magnification by $\mathbf{X}$. Because $\mathbf{X}$ affects different points differently the image is a distorted version of the object. $\mathrm{O}$ in Figure 2 is a closed curve through the points defined by vectors $\mathbf{x}_{i}$ in Figure 1. We can regard it as an object; the position vector of every point on it is magnified by $\mathbf{X}$ so the object itself is magnified by $\mathbf{X}$. The result is the image $I$ in Figure 2. $E_{2}$ and $E_{-2}$ are two lines along which there is magnification without rotation; they are defined by the eigenvectors of the magnification.

Notice that travelling around the circumference of $\mathrm{O}$, from $\mathbf{x}_{1}$ through $\mathbf{x}_{2}$ and then $\mathbf{x}_{3}, \mathbf{x}_{4}$ and $\mathbf{x}_{5}$ in order, takes one around the object in an anticlockwise sense while doing the same for corresponding points ( $\mathbf{y}_{1}, \mathbf{y}_{2}$, $\left.\mathbf{y}_{3}, \mathbf{y}_{5}, \mathbf{y}_{4}\right)$ takes one around the image in the clockwise sense. Generally speaking generalized magnification changes the shape, size and orientation of the object; and it may or may not change the (clockwise or anticlockwise) sense.

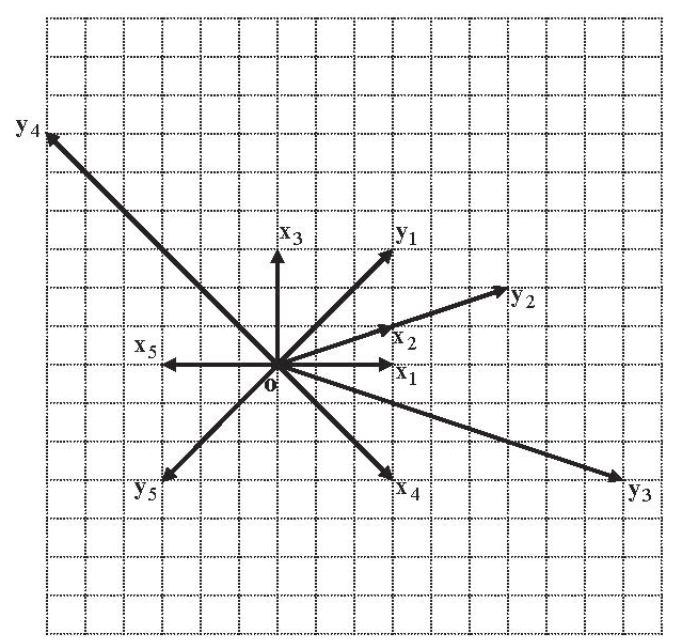

Figure 1 The effect of magnification $\mathbf{X}=\left(\begin{array}{cc}1 & 3 \\ 1 & -1\end{array}\right)$ on several

vectors. $\mathbf{o}$ is the origin; it is unaffected by the magnification. $\mathbf{x}_{1}$ is magnified to $\mathbf{y}_{1}, \mathbf{x}_{2}$ to $\mathbf{y}_{1}, \mathbf{x}_{3}$ to $\mathbf{y}_{3}$ and so on. Magnification by $\mathbf{X}$ changes the length and rotates most vectors. However $\mathbf{x}_{2}$ is doubled in length but is not rotated and $\mathbf{x}_{4}$ is doubled in length and its direction is reversed. $\mathbf{x}_{2}$ and $\mathbf{x}_{4}$ are eigenvectors of $\mathbf{X}$ and the corresponding eigenvalues are 2 and -2 . 
The generalized magnification in Figure 2 is defined by the matrix $\mathbf{X}$. But neither the matrix nor the figure give much insight into the nature of what has happened in the transformation of object to image. However, as the example suggests, a key to insight is the eigenstructure of the magnification.

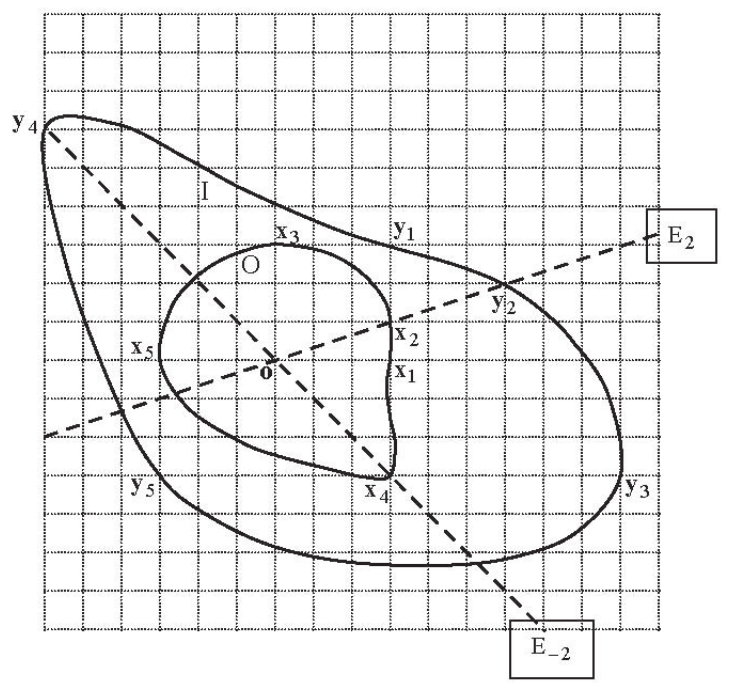

Figure $2 \mathrm{O}$ is the outline of an object on which the points represented by vectors $\mathbf{x}_{1}, \mathbf{x}_{2}, \mathbf{x}_{3}$, etc, lie. I is the outline of the image of the object having been magnified by magnification $\mathbf{X}=\left(\begin{array}{cc}1 & 3 \\ 1 & -1\end{array}\right)$; on it points represented by $\mathbf{y}_{1}, \mathbf{y}_{2}, \mathbf{y}_{3}$, etc, lie. Along $\mathrm{E}_{1}$ and $\mathrm{E}_{-2}$ there is magnification by 2 and -2 respectively and no rotation; 2 and -2 are the eigenvalues of $\mathbf{X}$ and $E_{1}$ and $\mathrm{E}_{-2}$ the corresponding eigenspaces.

\section{Eigenstructure of the magnification}

The eigenstructure of magnification $\mathbf{X}$ plays an important role in understanding what magnification in the generalized sense actually means. We turn to eigenstructure in general.

Consider an $n \times n$ real matrix $\mathbf{X}$. Suppose there exists a complex (that is, real or nonreal) scalar $\lambda$ and a nonnull complex vector $\mathbf{x}$ such that

$\mathbf{X x}=\lambda \mathbf{x}$.

Then $\lambda$ is called an eigenvalue of $\mathbf{X}$ and $\mathbf{x}$ is called an eigenvector of $\mathbf{X}$ corresponding to eigenvalue $\lambda \cdot{ }^{16-18}$

Informally what Equation 4 says is that, when it operates on certain vectors $\mathbf{x}$, matrix $\mathbf{X}$ is acting like a scalar; it multiplies $\mathbf{x}$ exactly as the scalar $\lambda$ multiplies $\mathbf{x}$. On other vectors $\mathbf{X}$ does not generally act like a scalar.
It follows from Equation 4 that if $\mathbf{x}$ happens to be an eigenvector of $\mathbf{X}$ then any scalar multiple of $\mathbf{x}$ is also an eigenvector of $\mathbf{X}$. All of the eigenvectors corresponding to a particular eigenvalue define what is called the eigenspace E corresponding to that eigenvalue. The eigenspaces corresponding to eigenvalues 2 and -2 in the example above are shown as $E_{2}$ and $\mathrm{E}_{-2}$, respectively, in Figure 2. In this case the eigenspaces are one-dimensional; they are straight lines and they correspond to what would be called principal meridians in optometry. We can refer to them as the eigenmeridians or principal meridians of the magnification $\mathbf{X}$. (It may seem a little odd that a line should be called a space but the description is perfectly logical. The line is formally a vector space and in other contexts the space may have three or more dimensions.)

Finding the eigenvalues and eigenspaces of a matrix is a classical problem in linear algebra; it is known as the eigenproblem or eigenvalue problem. Its solution is described in standard texts ${ }^{16-18}$ and given by matrix-handling software such as Matlab. The more interesting part of the eigenproblem is that of finding the eigenvalues. We shall summarize that part; for eigenvectors the interested reader is referred to the literature ${ }^{16-18}$.

The eigenvalues of a matrix $\mathbf{X}$ are the solutions $\lambda$ to the equation

$\operatorname{det}(\mathbf{X}-\lambda \mathbf{I})=0$.

The left-hand side of this equation is called the characteristic polynomial of $\mathbf{X}$ and the equation is called the characteristic equation of $\mathbf{X}$.

From here on we restrict attention to matrices $\mathbf{X}$ which are $2 \times 2$, the types that interest us. In terms of the entries of $\mathbf{X}$, then, the characteristic equation becomes

$\operatorname{det}\left(\left(\begin{array}{ll}x_{11} & x_{12} \\ x_{21} & x_{22}\end{array}\right)-\lambda\left(\begin{array}{ll}1 & 0 \\ 0 & 1\end{array}\right)\right)=0$

which multiplies out to

$\lambda^{2}-\lambda \operatorname{tr} \mathbf{X}+\operatorname{det} \mathbf{X}=0$.

It follows from the fundamental theorem of algebra $^{18}$ that $\mathbf{X}$ has two eigenvalues; let them be $\lambda_{-}$and 
$\lambda_{+}$. Solving the quadratic equation we obtain

$\lambda_{ \pm}=\operatorname{tr} \mathbf{X} \pm \sqrt{\operatorname{dis} \mathbf{X}}$

where

$$
\operatorname{dis} \mathbf{X}=(\operatorname{tr} \mathbf{X})^{2}-4 \operatorname{det} \mathbf{X}
$$

is the discriminant of $\mathbf{X}$.

Equation 8 reveals three possible situations: If $\operatorname{dis} \mathbf{X}>0$ then $\lambda_{-}$and $\lambda_{+}$are real and distinct; if $\operatorname{dis} \mathbf{X}=0$ then $\lambda_{-}$and $\lambda_{+}$are real but the same; and if $\operatorname{dis} \mathbf{X}<0$ then $\lambda_{-}$and $\lambda_{+}$are complex and not real. For the numerical example above $\lambda_{-}=-2$ and $\lambda_{+}=2$.

The discriminant can also be written as

$$
\operatorname{dis} \mathbf{X}=4\left(X_{\mathrm{J}}^{2}+X_{\mathrm{K}}^{2}-X_{\mathrm{L}}^{2}\right)
$$

where $X_{\mathrm{J}}, X_{\mathrm{K}}$ and $X_{\mathrm{L}}$ are the coefficients in the expansion

$$
\mathbf{X}=X_{\mathrm{I}} \mathbf{I}+X_{\mathrm{J}} \mathbf{J}+X_{\mathrm{K}} \mathbf{K}+X_{\mathrm{L}} \mathbf{L} .
$$

$\mathbf{I}, \mathbf{J}, \mathbf{K}$ and $\mathbf{L}$ are the same basic matrices used elsewhere for dioptric power and other $2 \times 2$ optical properties $^{20,21}$.

Instead of $\lambda_{ \pm}$we shall write the eigenvalues of $\mathbf{X}$ as $X_{ \pm}$. They can be called the principal magnifications or eigenmagnifications of the magnification $\mathbf{X}$. In fact it is useful to write magnifications in principal meridional form or eigenform as $X_{-}\left\{A_{-}\right\} X_{+}\left\{A_{-}\right\}$ just as we do for dioptric power 22 . The $A_{ \pm}$are angles representing the corresponding eigenspaces; they are the principal meridians of the magnification. We mention also that ${ }^{16}$

$$
\operatorname{det} \mathbf{X}=X_{-} X_{+} \text {. }
$$

Equation 8 shows that $X_{-} \leq X_{+}$when they are real numbers. We shall also write the principal magnifications as $X_{1}$ and $X_{2}$ where order on the number line is not implied.

Expressed in principal meridional form the magnification of the numerical example above is $-2\{135.0\} 2\{18.4\}$.

As Figure 2 illustrates generalized magnification, in forming images, typically magnifies and distorts and rotates, reflects or inverts objects. There are spe- cial cases in which the magnification reduces the object to a line segment or to a point. What happens in any given case depends on the rank of the matrix $\mathbf{X}$, the topic to which we now turn.

\section{Rank of the magnification}

The values of $\mathbf{x}$ in Equation 3 may be restricted by the geometry of the physical structure such as an aperture for example. However, we shall not address that issue here. What interests us is the restriction on the possible values of $\mathbf{y}$ that arise out of the mathematical nature of the magnification $\mathbf{X}$ itself. The set of possible values of $\mathbf{y}$ is called the range of $\mathbf{X} .{ }^{17}$

It is obvious from Equation 3 that, if $\mathbf{X}=\mathbf{O}$, then $\mathbf{y}=\mathbf{o}$ regardless of the value of $\mathbf{x}$; thus the range of $\mathbf{X}$ is zero-dimensional. If $\mathbf{X}$ is singular but not null then it turns out that $\mathbf{y}$ lies on a straight line through $\mathbf{0}$; the range of $\mathbf{X}$ then is one-dimensional. If $\mathbf{X}$ is nonsingular then the range of $\mathbf{X}$ is two-dimensional. In these three cases $\mathbf{X}$ is said to have rank 0,1 and 2 respectively ${ }^{17}$.

The rank of $\mathbf{X}$, then, defines the dimensionality of the image. (In Matlab it is given by the function rank.) If $\mathbf{X}$ has rank 0 the image is a point; if the rank is 1 then the image is confined to a straight line; if the rank is 2 then the image may occupy any part of the plane. (Examples will be seen in Figure 4 below.)

Below we shall interpret magnifications in terms of combinations of simpler magnifications. To do so we shall need to know how magnifications combine.

\section{Successive magnification}

Suppose magnification $\mathbf{X}_{1}$ is followed by magnification $\mathbf{X}_{2}$. By Equation 3 the initial magnification magnifies $\mathbf{x}$ to $\mathbf{y}$ according to

$\mathbf{X}_{1} \mathbf{x}=\mathbf{y}$.

Also by Equation 3 the second magnification magnifies $\mathbf{y}$ to $\mathbf{z}$ according to

$\mathbf{X}_{2} \mathbf{y}=\mathbf{z}$.

Substituting from Equation 13 into Equation 14 we obtain

$\mathbf{X}_{2} \mathbf{X}_{1} \mathbf{x}=\mathbf{z}$.

It follows that the overall effect is to magnify $\mathbf{x}$ to $\mathbf{z}$ according to 
$\mathbf{X x}=\mathbf{z}$

where

$$
\mathbf{X}=\mathbf{X}_{2} \mathbf{X}_{1}
$$

Equation 17 provides the formula for successive magnifications needed below. tion.

We now examine particular types of magnifica-

\section{Scalar magnification}

Suppose magnification $\mathbf{X}$ has the form

$$
\mathbf{X}=X \mathbf{I} \text {. }
$$

Matrix $\mathbf{X}$ is then called a scalar matrix and we shall refer to the magnification as scalar magnification. It is a negative, nonnegative or positive scalar magnification according as $X<0, X \geq 0$ or $X>0$. If $X=0$ it is null magnification and if $X=1$ it is identity magnification or identification. Equation 3 becomes

$X \mathbf{x}=\mathbf{y}$

because $\mathbf{I x}=\mathbf{x}$. Thus every vector $\mathbf{x}$ is simply multiplied by the scalar $X$; the matrix $\mathbf{X}$ behaves as a scalar for all vectors.

The discriminant of $\mathbf{X}$ (Equation 10) is zero and $X$ is the only eigenvalue (Equation 8). Equation 4 shows that every vector $\mathbf{x}$ is an eigenvector corresponding to eigenvalue $X$. The corresponding eigenspace is twodimensional. Every meridian is a principal or eigenmeridian. The determinant of $\mathbf{X}$ is $X^{2}$ and, hence, the rank of $\mathbf{X}$ is 2 . The only exception is for null magnification for which the rank is 0 .

Examples of scalar magnification are shown in Figure 3 for scalars $X=-2,-1,-1 / 2,0,1 / 2,1,2$.

For $X=1$ (that is, $\mathbf{X}=\mathbf{I}$ ) the image is the same as the object (identification).

For $X=2$ every point $\mathbf{x}$ becomes point $\mathbf{y}=2 \mathbf{x}$; its distance from the origin is doubled. There are no rotational effects. The object gets stretched outward equally in all directions. $\mathbf{X}=2 \mathbf{I}$ represents what is called two-times magnification in conventional usage.

$\mathbf{X}=\frac{1}{2} \mathbf{I}$ represents scalar magnification by the factor $\frac{1}{2}$. Magnification minifies.
Scalar magnification $\mathbf{X}=-\mathbf{I}$ reverses the position of every point on the object relative to the origin; there is inversion along all meridians through the origin. Notice that the image is not simply up-side down. There is in fact nothing special about the directions up and down; the same thing happens in all meridians. The image is as much left-side right as it is up-side down. Also notice that the arrow of the object points anti-clockwise and so does the arrow of the image. We say that the magnification preserves the sense of the object. Inversion is one way of describing magnification $\mathbf{- I}$; another is that it is a rotation of the object through $180^{\circ}$, or a half-turn, about an axis perpendicular to the plane. Indeed the description of -I as a rotation seems to be the clearer; we regard it, therefore, as the preferred description of magnification $-\mathbf{I}$.

Negative and positive scalar magnifications all preserve the sense of the object.

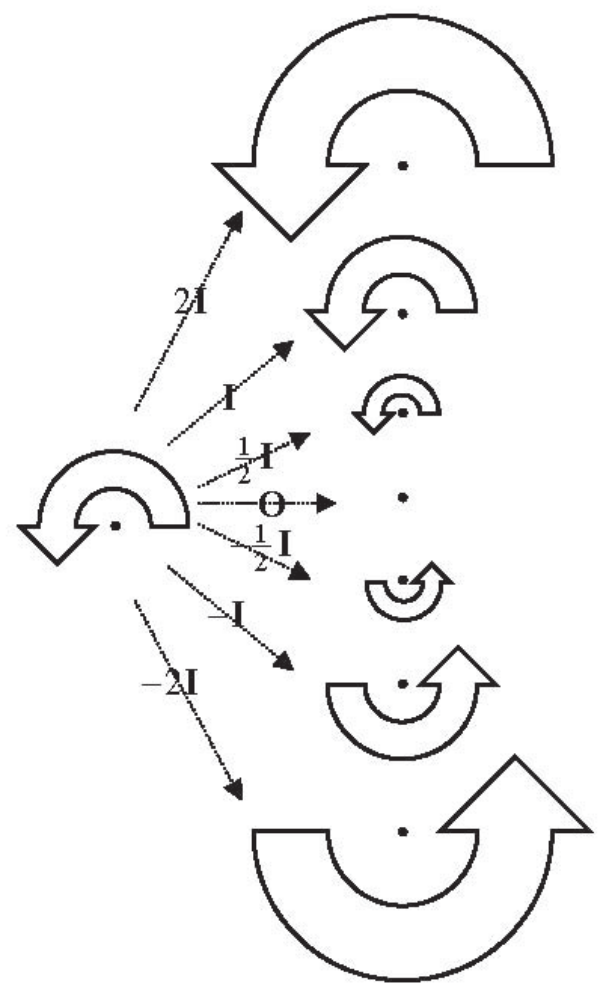

Figure 3 Some scalar magnifications. Identity magnification I leaves the object unchanged. Null magnification $\mathbf{O}$ reduces the object to a point. Magnification 2I doubles the dimensions of the object without changing its shape and orientation; it is equivalent to what is commonly called two-times magnification. Negative scalar magnification changes the size of the object and rotates it through $180^{\circ}$.

The negative scalar magnification $-2 \mathbf{I}$ can be 
written as $(-\mathbf{I})(2 \mathbf{I})$. Hence, by Equation 17, it can be regarded as a positive scalar magnification (2I) followed by a half turn $(-\mathbf{I})$. In general any negative scalar magnification $X \mathbf{I}$ can be viewed as a positive scalar magnification $-X \mathbf{I}$ followed by a half-turn. The order of the two magnifications can be reversed (half-turn followed by positive scalar magnification) though this is not possible for magnifications in general because of noncommutativity of matrix multiplication in Equation 17.

Scalar magnification is what one has in systems with no astigmatic elements. More interesting for us is magnification in systems with astigmatic elements. Symmetric magnification comes as no surprise.

\section{Symmetric magnification}

Suppose $\mathbf{X}$ is symmetric and nonscalar. The magnification itself is then called symmetric. The antisymmetric coefficient $X_{\mathrm{L}}$ in Equation 11 is zero and the discriminant (Equation 10) is always positive. It follows from Equation 8 that the eigenvalues are always real. Just as for symmetric dioptric power the principal meridians of magnification are orthogonal. ${ }^{23}$ In effect we have scalar magnification along the two eigenmeridians and we can draw magnification crosses just as we draw power crosses for dioptric power.

Figure 4 illustrates the effects of the magnifications listed in Table 1 on a particular object $O$. (f) and (p) are the images under identification $(\mathbf{X}=\mathbf{I})$ and null magnification $(\mathbf{X}=\mathbf{O})$; they both represent scalar magnifications. (m) also represents a scalar magnification, negative scalar magnification $(\mathbf{X}=-\mathbf{I})$. The rest represent nonscalar symmetric magnifications with the same principal meridians.

Image $(\mathrm{g})$ in Figure 4 represents the magnification $2\{30\} 1\{120\}$; the object has been stretched to twice its length along the $30^{\circ}$-meridian while is length in the $120^{\circ}$-meridian has not been changed. In (e) (magnification $0.5\{30\} 1\{120\}$ ) the object's length has been halved along the $30^{\circ}$-meridian; there is minification along that meridian. In (d) the magnification along the $30^{\circ}$-meridian is zero; the object has been reduced to a line segment along the $120^{\circ}$-merid- ian. The magnification $(0\{30\} 1\{120\})$ in this case is singular (the determinant is $0 \times 1$ ) and the rank 1 .

By analogy with dioptric power magnification $-0.5\{30\} 1\{120\}$ might be called mixed. The image is (c) in Figure 4. There has been minification (by half) and inversion (the negative sign) along the $30^{\circ}$-meridian while again there has been no change along the $120^{\circ}$-meridian. (b) $(-1\{30\} 1\{120\})$ represents pure inversion along $30^{\circ}$-meridian with no change in length; one might refer to this as an example of anti-scalar magnification. In (a) ( -2 . $\{30\} 1\{120\}$ ) there has been inversion and magnification along the $30^{\circ}$-meridian.

Images (a), (b), (c), (d), (e) and (g) are all under magnification with unit principal magnification along the $120^{\circ}$-meridian. (h), (i), (j) and (k) are images under unit magnification along the $30^{\circ}$-meridian. The rest of the images in Figure 4 represent magnifications of which neither principal magnification is 1. (n) and (j) represent singular magnifications (rank 1).

Notice that magnification preserves the anticlockwise orientation of the object in Figure 4 in those cases whose principal magnifications have the same sign or, equivalently, have determinant greater than 0 . On the other hand magnifications whose principal magnifications differ in sign (by Equation 12 the determinant is less than 0 ) reverse the sense to become clockwise.

A symmetric matrix is called positive semidefinite if none of its eigenvalues is less than zero. ${ }^{17,23}$ The magnification can also be called positive semidefinite. (d), (e), (f), (g), (j), (k) and (p) in Figure 4 and Table 1 represent positive semidefinite magnifications. Positive semidefinite magnifications seems relatively easy to visualize. As we shall see below symmetric magnifications that are not positive semidefinite can be interpreted as combinations of positive semidefinite magnifications and rotations or reflections.

There is a special class of symmetric magnifications that cause reflection. We leave discussion of them until after we have examined magnifications that are rotations.

We turn attention now to magnifications that are not symmetric. 


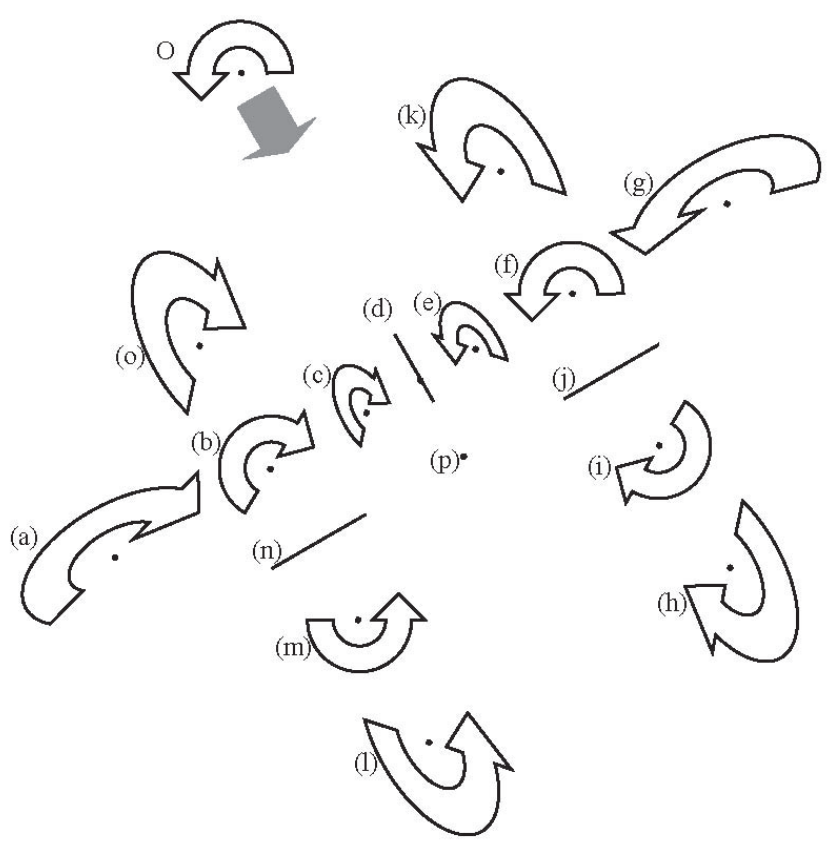

Figure 4 Some symmetric magnifications of object O. (a) to (p) are images under the magnifications. The corresponding magnifications are listed in principal meridional form and as matrices

in Table 1. $\mathbf{X}_{\mathrm{f}}=\mathbf{I}$ and $\mathbf{X}_{\mathrm{m}}=-\mathbf{I} . \mathbf{X}_{\mathrm{p}}=\mathbf{O}$ is a magnification of rank $0 ; \mathbf{X}_{\mathrm{j}}, \mathbf{X}_{\mathrm{d}}$ and $\mathbf{X}_{\mathrm{n}}$ are magnifications of rank 1; the rest are magnifications of rank 2. Notice that the sense of the arrow in images (e), (f), (g), (k), (l) and (m) is anticlockwise (the same as in O) but clockwise in images (a), (b), (c), (h), (i) and (o).

\section{Asymmetric magnification}

If $\mathbf{X}$ is not symmetric the magnification is asymmetric. The numerical example above (and illustrated in Figures 1 and 2) is a case in point. It is an example of an asymmetric magnification with positive discriminant and, because of Equation 8, it has real and distinct principal magnifications. There are two other classes of asymmetric magnification: those with discriminant 0 and those with discriminant less than 0 .

An example of asymmetric magnification with zero discriminant is $\mathbf{X}=\left(\begin{array}{ll}0 & 1 \\ 0 & 0\end{array}\right)$. From the zeros in the bottom row one sees that the image of any object under this magnification will have zero length in the vertical direction. However the zeros in the first column mean that the horizontal length of the object is reduced to nothing. In effect this magnification magnifies any object to nothing in the horizontal direction and then rotates it clockwise through $90^{\circ}$. (We exam- ine this magnification more fully below.) An example is shown in Figure 5. Notice how this differs from the magnification represented by Figure 4(d) where there is no rotation. Now there is a single eigenvalue, namely 0 , as one sees from Equation 8. The (only) eigenmeridian is horizontal.

In cases of this type the principal meridional representation of magnification breaks down just as it does

Table 1 Symmetric magnifications of object $\mathrm{O}$ in Figure 4.

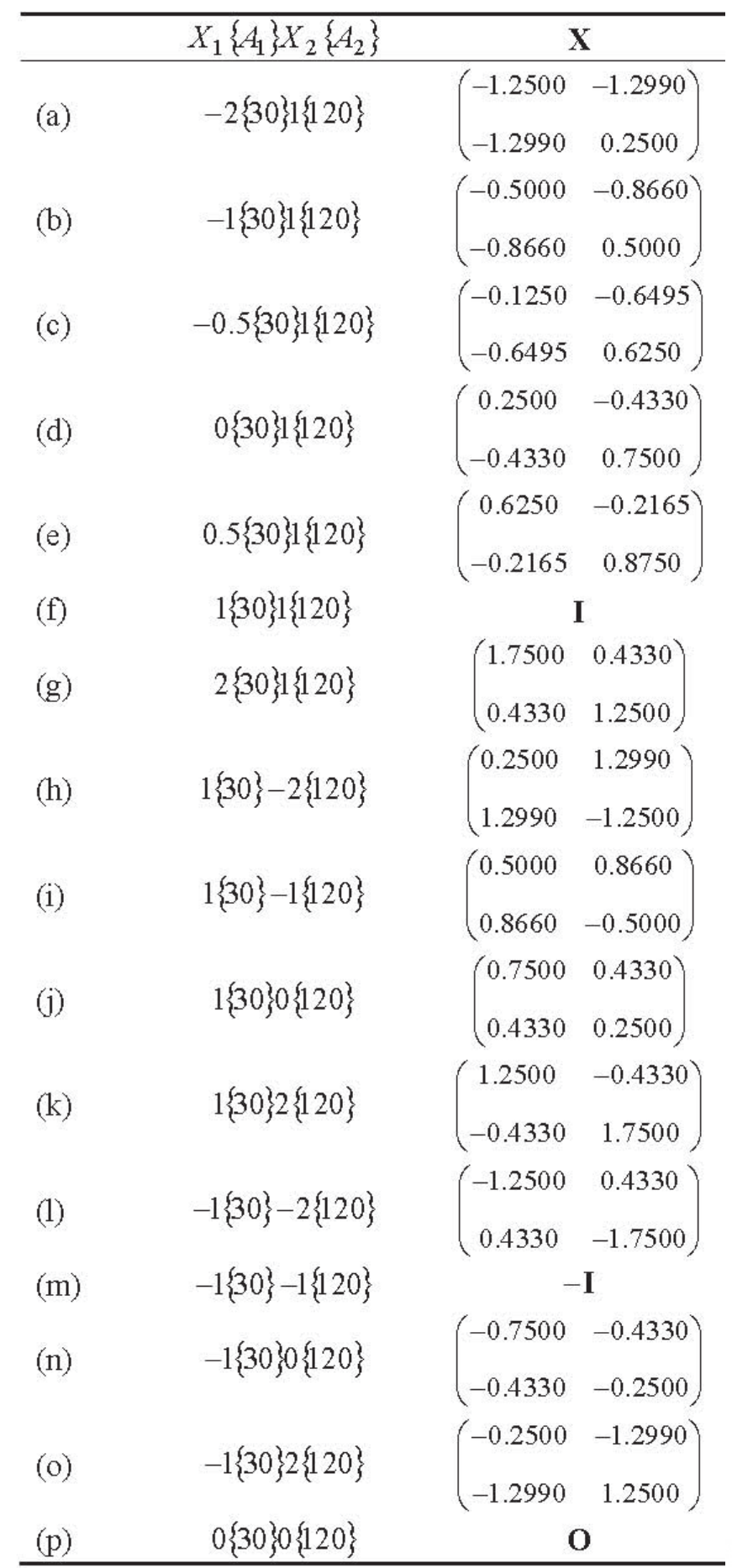


in the corresponding situation for dioptric power 22 ; on the other hand the matrix representation of magnification as $\mathbf{X}$ works always.

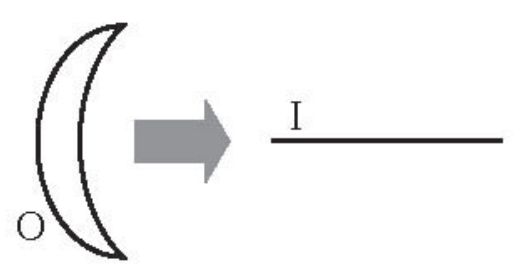

Figure 5 The image I of an object $\mathrm{O}$ under the singular asymmetric magnification $\mathbf{X}=\left(\begin{array}{ll}0 & 1 \\ 0 & 0\end{array}\right)$. The magnification can be interpreted as symmetric magnification $0\{180\} 1\{90\}$ followed by clockwise rotation through $90^{\circ}$.

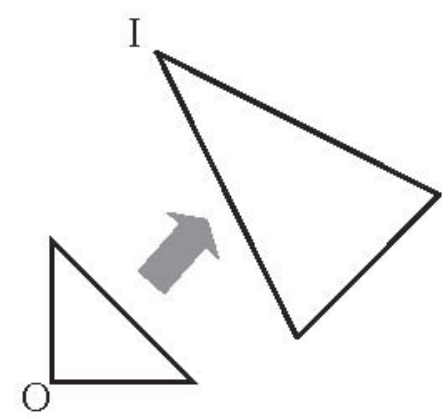

Figure 6 The image I of an object $\mathrm{O}$ under asymmetric magnification $\left(\begin{array}{cc}1 & 2 \\ -2 & -1\end{array}\right)$. The magnification is equivalent to symmetric magnification $1\{135\} 3\{45\}$ followed by clockwise rotation through $90^{\circ}$.

Magnifications with discriminants less than zero have nonreal eigenvalues and nonreal eigenvectors; there are no actual principal meridians. Nevertheless it is still meaningful to write the magnification as $X_{-}\left\{A_{-}\right\} X_{+}\left\{A_{-}\right\}$although none of the four numbers is real. An example is the magnification by $\left(\begin{array}{cc}1 & 2 \\ -2 & -1\end{array}\right)$ illustrated in Figure 6. The principal meridional magnification is $-1.732 i\{135+37.7 i\} 1.732 i\{135-37.7 i\}$, the principal magnifications being imaginary.

The most important asymmetric magnifications for our purposes, however, are pure rotations. We examine them next.

\section{Magnification as rotation}

Consider the magnification

$\mathbf{X}=\mathbf{R}_{\theta}$

where

$$
\mathbf{R}_{\theta}=\left(\begin{array}{cc}
\cos \theta & -\sin \theta \\
\sin \theta & \cos \theta
\end{array}\right)
$$

is a rotation matrix ${ }^{10,23,24}$; it represents anticlockwise rotation through angle $\theta$. It has

$\operatorname{dis} \mathbf{X}=4\left((\cos \theta)^{2}-1\right)$

This type of generalized magnification represents pure rotation. When $\theta=0^{\circ}$ the rotation reduces to identification ( $\mathbf{X}=\mathbf{I}$ ). When $\theta=180^{\circ}$ the rotation is equivalent to the negative scalar magnification $-\mathbf{I}$ as illustrated in Figures 3 and 4(m). In other cases $\mathbf{X}$ is

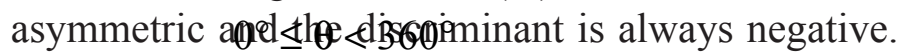
We consider

Some examples of magnifications that are rotations are shown in Figure 7. The determinant of the magnification is 1 and the anticlockwise sense of the object is preserved under the magnification in all cases.

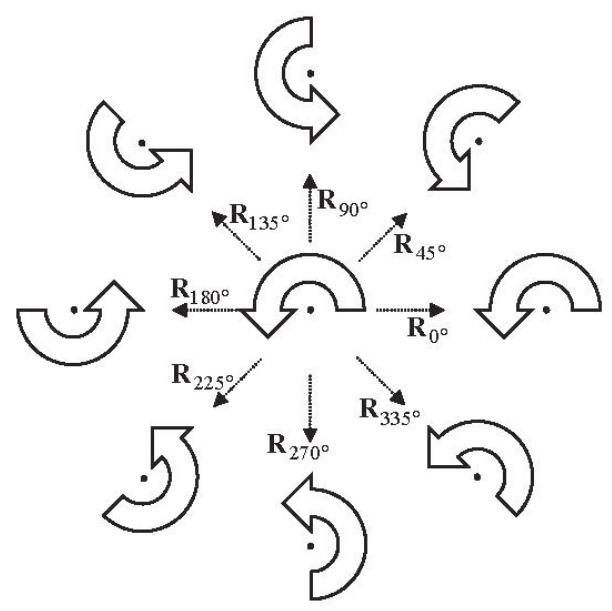

Figure 7 Some rotational magnifications. Note that the anticlockwise sense of the arrow in the object is preserved in the images. $\mathbf{R}_{0^{\circ}}=\mathbf{I}$ and $\mathbf{R}_{180^{\circ}}=-\mathbf{I}$. 


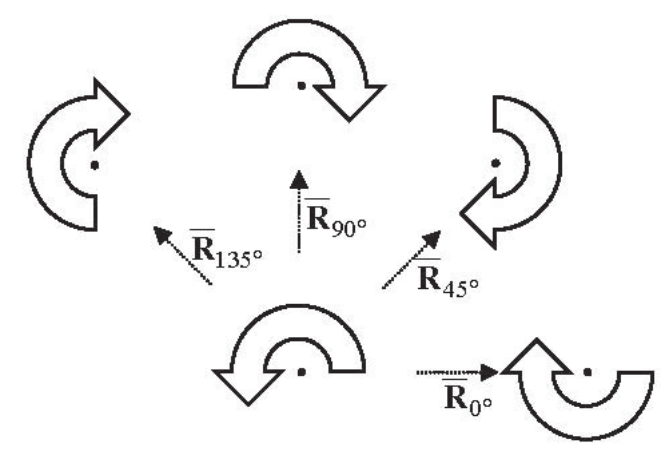

Figure 8 Some reflectional magnifications. Note that the anticlockwise sense of the arrow in the object is reversed in the images.

For later reference we note that

$$
\mathbf{R}_{\theta}^{\mathrm{T}}=\mathbf{R}_{\theta}^{-1}=\mathbf{R}_{-\theta}
$$

where T represents the matrix transpose.

It is natural next to examine generalized magnifications that are reflections; they are actually examples of symmetric magnifications.

\section{Magnification as reflection}

Now consider generalized magnification

$$
\mathbf{X}=\overline{\mathbf{R}}_{\theta}
$$

where

$\overline{\mathbf{R}}_{\theta}=\left(\begin{array}{cc}\cos 2 \theta & \sin 2 \theta \\ \sin 2 \theta & -\cos 2 \theta\end{array}\right)$.

$\overline{\mathbf{R}}_{\theta}$ is a reflection matrix ${ }^{10,24}$; it represents reflection in the meridian at angle $\theta$. Examples are shown in Figure $8 . \overline{\mathbf{R}}_{0^{\circ}}$ is a reflection in the horizontal meridian; it is a vertical inversion. $\overline{\mathbf{R}}_{90^{\circ}}$ is a horizontal inversion. Obviously $\overline{\mathbf{R}}_{180^{\circ}}=\overline{\mathbf{R}}_{0^{\circ}}$. We need consider $0^{\circ} \leq \theta<180^{\circ}$.

The determinant of a reflection is -1 ; the anticlockwise sense of the object in Figure 8 is reversed in all the reflected images. We note that

$\overline{\mathbf{R}}_{\theta}^{-1}=\overline{\mathbf{R}}_{\theta}$.

(A matrix like this whose inverse is itself is called involutory. ${ }^{16,25}$ )
With a view to interpreting asymmetric magnifications and symmetric magnifications that are not positive semidefinite we turn now to combinations of symmetric magnifications on one hand and rotations and reflections on the other.

\section{Magnification as combination of symmetric mag- nification and rotation}

Suppose symmetric magnification $\mathbf{X}_{\mathrm{sym}}$ is followed by rotation $\mathbf{R}_{\theta}$. It follows from Equation 17 that the result is magnification $\mathbf{X}$ given by

$\mathbf{X}=\mathbf{R}_{\theta} \mathbf{X}_{\text {sym }}$.

We now pose the following question: Given an asymmetric magnification $\mathbf{X}$ is it possible to treat it as a symmetric magnification followed by a rotation? This amounts to asking whether one can factorize $\mathbf{X}$ into post- and pre-factors $\mathbf{X}_{\text {sym }}$ and $\mathbf{R}_{\theta}$.

Let us write Equation 27 as

$\mathbf{X}=\left(\begin{array}{cc}c & -s \\ s & c\end{array}\right)\left(\begin{array}{ll}a & b \\ b & d\end{array}\right)$.

Hence

$\mathbf{X}=\left(\begin{array}{cc}c a-s b & c b-s d \\ s a+c b & s b+c d\end{array}\right)$

Thus

$X_{\mathrm{I}}=c(a+d) / 2$

and

$X_{\mathrm{L}}=-s(a+d) / 2$.

(This corresponds to determining the coefficients $F_{\mathrm{I}}$ and $F_{\mathrm{L}}$ of the dioptric power matrix $\mathrm{F}^{21}$ ) But $\tan \theta=s / c$. Hence

$\tan \theta=-X_{\mathrm{L}} / X_{\mathrm{I}}$.

From Equations 23 and 27 we have

$\mathbf{X}_{\mathrm{sym}}=\mathbf{R}_{\theta}^{\mathrm{T}} \mathbf{X}$.

It follows that for a given magnification $\mathbf{X}$ we can calculate $\theta$ by means of Equation 32, $\mathbf{R}_{\theta}$ by means 
of Equation 21 and, finally, $\mathbf{X}_{\text {sym }}$ by means of Equation 33. For $\theta$ there are two solutions in $\left[0^{\circ}, 360^{\circ}\right)$ and, hence, two symmetric magnifications. But the solutions differ by $180^{\circ}$ and so the one rotation matrix is the negative of the other. Hence the one symmetric magnification is the negative of the other. This procedure works for all asymmetric magnifications. Formally Equation 32 fails when $X_{\mathrm{I}}=0$ but then one puts $\theta=90^{\circ}$ or $270^{\circ}$.

This procedure of factorizing a magnification into a symmetric magnification followed by a rotation also works for symmetric magnifications $\left(X_{\mathrm{L}}=0\right)$; then, from Equation $32, \theta=0^{\circ}$ or $180^{\circ}$. That is so, however, provided $X_{\mathrm{I}} \neq 0$. If $X_{\mathrm{I}}=0$ the right-hand side of Equation 32 is indeterminate and the procedure fails.

For the numerical example above $X_{\mathrm{I}}=0$ and $X_{\mathrm{L}}=1$. So $\theta=90^{\circ}$ or $270^{\circ}$. In the first case Equation 21 becomes

$\mathrm{R}_{90^{\circ}}=\left(\begin{array}{cc}0 & -1 \\ 1 & 0\end{array}\right)=-\mathbf{L}$

and from Equation 33

$$
\mathbf{X}_{\mathrm{sym}}=\left(\begin{array}{cc}
0 & 1 \\
-1 & 0
\end{array}\right)\left(\begin{array}{cc}
1 & 3 \\
1 & -1
\end{array}\right)=\left(\begin{array}{cc}
1 & -1 \\
-1 & -3
\end{array}\right) \text {. }
$$

The symmetric part of the magnification has principal meridional magnification $-3.24\{76.7\} 1.24\{166.7\}$. Thus the asymmetric magnification of the numerical example can be thought of as symmetric magnification $-3.24\{76.7\} 1.24\{166.7\}$ followed by anticlockwise rotation by $90^{\circ}$. Alternatively, choosing $\theta=270^{\circ}$ we find that the magnification can also be interpreted as symmetric magnification $3.24\{76.7\}-1.24\{166.7\}$ followed by anticlockwise rotation by $270^{\circ}$ or, equivalently, clockwise rotation by $90^{\circ}$.

It follows from the discussion above that every magnification $\mathbf{X}$, with the exception of symmetric magnifications with $X_{\mathrm{I}}=0$, can be regarded as symmetric magnification followed by a rotation. This can be done in two ways.

We now consider magnifications that are combinations of symmetric magnifications and reflections.

\section{Magnification as combination of symmetric mag- nification and reflection}

Can a magnification $\mathbf{X}$ be regarded as a symmetric magnification followed by a reflection? The question asks whether $\mathbf{X}$ can be factorized as

$$
\mathbf{X}=\overline{\mathbf{R}}_{\theta} \mathbf{X}_{\text {sym }}
$$

Proceeding much as for rotations we find that

$\tan 2 \theta=X_{\mathrm{K}} / X_{\mathrm{J}}$.

This gives a reflection $\overline{\mathbf{R}}_{\theta}$ in terms of the coefficients of $\mathbf{X}$ in Equation 11. Because of Equations 26 and 35 we find that

$\mathbf{X}_{\mathrm{sym}}=\overline{\mathbf{R}}_{\theta} \mathbf{X}$.

It follows that any magnification $\mathbf{X}$ can be regarded as a symmetric magnification $\mathbf{X}_{\text {sym }}$ followed by a reflection $\overline{\mathbf{R}}_{\theta}$ where $\theta$ is given by Equation 36 and $\mathbf{X}_{\text {sym }}$ by Equation 37. Again there are two solutions for $\theta$, but now they are $90^{\circ}$ apart. One can calculate $\mathbf{X}_{\text {sym }}$ corresponding to one of them and simply reverse the sign for $\mathbf{X}_{\text {sym }}$ corresponding to the other. For magnifications with $X_{\mathrm{J}}=0$ we must put $2 \theta=90^{\circ}$ or $270^{\circ}$, that is, $\theta=45^{\circ}$ or $135^{\circ}$. The interpretation fails, however, for magnifications with both $X_{\mathrm{J}}=0$ and $X_{\mathrm{K}}=0$.

For the numerical example above one obtains $\theta=31.7^{\circ}$ or $121.7^{\circ}$. For $\theta=31.7^{\circ}$

$\mathbf{X}_{\mathrm{sym}}=\left(\begin{array}{ll}1.3416 & 0.4472 \\ 0.4472 & 3.1305\end{array}\right)$

which is equivalent to the positive semidefinite magnification $1.24\{166.7\} 3.24\{76.4\}$. Choosing $\theta=121.7^{\circ}$ gives the principal magnification $-1.24\{166.7\}-3.24\{76.4\}$.

Thus every magnification can be viewed as a symmetric magnification followed by a reflection, also in two ways. The only magnifications for which this is not so are those with $X_{\mathrm{J}}=X_{\mathrm{K}}=0$. 


\section{Interpreting generalized magnification}

The asymmetric magnification in the numerical example above has been decomposed into a symmetric magnification followed by a rotation or a reflection; two angles are possible for the rotation and two for the reflection. Of the four possibilities only one has the principal magnifications of the symmetric magnification both positive; it is the positive semidefinite (positive definite in fact) symmetric magnification $1.24\{166.7\} 3.24\{76.4\}$ followed by the reflection $\overline{\mathbf{R}}_{31.4^{\circ}}$. This would seem to be the preferred interpretation.

A magnification $\mathbf{X}$ can be interpreted as a positive semidefinite magnification followed by a rotation or a reflection according as its determinant is nonnegative or negative. This follows because the determinant of $\mathbf{X}_{\text {sym }}$ equals the determinant of $\mathbf{X}$ in the case of Equation 33 but the negative of the determinant of $\mathbf{X}$ in the case of Equation 37.

We are led to the following procedure for interpreting a generalized magnification $\mathbf{X}$. If the magnification is positive semidefinite we describe it as such. That includes symmetric magnifications with nonnegative principal magnifications and positive scalar magnifications, identification and null magnification. If the magnification is not positive semidefinite we choose rotation if $\operatorname{det} \mathbf{X} \geq 0$ and reflection otherwise. Two angles $\theta$ are determined as described above in the case of combinations with a rotation and two as described above for combinations with a reflection. $\mathbf{X}_{\text {sym }}$ is then calculated for each angle according to Equation 33 (rotation) or Equation 37 (reflection). Of the two angles we select that one which makes $\mathbf{X}_{\text {sym }}$ positive semidefinite.

When $X_{\mathrm{I}}=X_{\mathrm{L}}=0$ a rotation cannot be found and when $X_{\mathrm{J}}=X_{\mathrm{K}}=0$ a reflection cannot be found. However, in the former case $\operatorname{det} \mathbf{X}<0$ which requires a reflection while in the latter case $\operatorname{det} \mathbf{X} \geq 0$ which requires a rotation. Thus the procedure always works.

The procedure described here illustrates the general principle known in linear algebra as polar decomposition $^{25}$. Matrix $\mathbf{X}$ can be decomposed as

$$
\mathbf{X}=\mathbf{R} \mathbf{X}_{\text {sym }}
$$

(compare Equations 27 and 35) where

$$
\mathbf{X}_{\text {sym }}=\left(\mathbf{X}^{\mathrm{T}} \mathbf{X}\right)^{1 / 2}
$$

is positive semidefinite and

$$
\mathbf{R}=\mathbf{X} \mathbf{X}_{\mathrm{sym}}^{-1}
$$

is orthogonal ( $\mathbf{R}_{\theta}$ or $\overline{\mathbf{R}}_{\theta}$ ).

The interpretation of generalized magnification is now illustrated by means of a few numerical examples.

\section{More numerical examples}

Magnification $\mathbf{O}$ is null magnification. It is symmetric and its principal magnifications (both 0) are nonnegative. Thus null magnification is positive semidefinite and, hence, we interpret it simply as null magnification.

Identification $\mathbf{I}$ is also positive semidefinite; we interpret it simply as identification. The same is true of all positive scalar magnifications.

Magnification -I is not positive semidefinite. (It is negative definite in fact.) Hence we interpret it as a symmetric magnification followed by either a rotation or a reflection. Because its determinant is positive we choose rotation. $X_{\mathrm{L}}=0$ and $X_{\mathrm{I}}=-1$ and, hence, from Equation $32 \tan \theta=0$. Thus we have the two angles $\theta=0^{\circ}$ or $180^{\circ}$ and the two possible rotations $\mathbf{R}_{0^{\circ}}=\mathbf{I}$ and $\mathbf{R}_{180^{\circ}}=-\mathbf{I}$ as follows from Equation 21. Equation 33 gives the two corresponding symmetric magnifications $-\mathbf{I}$ and $\mathbf{I}$. Only the latter is positive semidefinite. Hence we have the interpretation of magnification - I as magnification I (identification) followed by anticlockwise rotation through $180^{\circ}$. (Of course 'anticlockwise' is redundant here.) This interpretation is in keeping with that already mentioned in connection with Figure 3.

$$
\text { Consider the magnification }\left(\begin{array}{ll}
1 & 0 \\
0 & 0
\end{array}\right) \text {. It has rank }
$$

1 and so an image is confined to a straight line. In principal meridional form the magnification is 
$1\{180\} 0\{90\}$. The magnification is positive semidefinite, so we interpret it as it is: magnification reduces the vertical width of the object to zero and leaves the horizontal width unchanged.

The magnification $\left(\begin{array}{cc}1 & 0 \\ 0 & -1\end{array}\right)$ is $\mathbf{J}$ or, equivalently, $1\{180\}-1\{90\}$. This is symmetric but not positive semidefinite. (It is indefinite.) Because the determinant is negative we choose reflections. (This is a case in which we could not choose rotations anyway because $X_{\mathrm{I}}=X_{\mathrm{L}}=0$.) $X_{\mathrm{K}}=0$ and $X_{\mathrm{J}}=1$ and, hence, $\tan 2 \theta=0$ by Equation 36. Thus $2 \theta=0^{\circ}$ or $180^{\circ}$. Hence $\theta=0^{\circ}$ or $90^{\circ}$. The corresponding reflections are $\overline{\mathbf{R}}_{0^{\circ}}=\left(\begin{array}{cc}1 & 0 \\ 0 & -1\end{array}\right)=\mathbf{J}$ and $\overline{\mathbf{R}}_{90^{\circ}}=-\mathbf{J}$. Hence we obtain the corresponding symmetric magnifications $\mathbf{J J}=\mathbf{I}$ and $-\mathbf{J J}=-\mathbf{I}$ by Equation 37 . The former is positive semidefinite and so we interpret $\mathbf{J}$ as identification I followed by reflection $\overline{\mathbf{R}}_{0}$ in the horizontal or, simply, reflection in the horizontal (see Figure 8).

The singular magnification $\left(\begin{array}{ll}0 & 1 \\ 0 & 0\end{array}\right)$ is asymmetric. Because the determinant is 0 we choose rotation. $X_{\mathrm{I}}=0$ and $X_{\mathrm{L}}=0.5$. Thus $\theta=90^{\circ}$ or $270^{\circ}$. By Equation 33 we obtain the symmetric magnifications $\mathbf{X}_{\mathrm{sym}}=\left(\begin{array}{cc}0 & 1 \\ -1 & 0\end{array}\right)\left(\begin{array}{ll}0 & 1 \\ 0 & 0\end{array}\right)=\left(\begin{array}{cc}0 & 0 \\ 0 & -1\end{array}\right)$

and

$$
\mathbf{X}_{\mathrm{sym}}=\left(\begin{array}{ll}
0 & 0 \\
0 & 1
\end{array}\right)
$$

respectively. The latter represents $1\{180\} 0\{90\}$ and, so, is positive semidefinite. (The former is negative semidefinite.) Thus the magnification is equivalent to $1\{180\} 0\{90\}$ followed by anticlockwise rotation through $270^{\circ}$ or, equivalently, clockwise rotation through $90^{\circ}$ as already illustrated in Figure 5.

$$
\text { Magnification }\left(\begin{array}{cc}
1 & 2 \\
-2 & -1
\end{array}\right) \text { has positive determi- }
$$

nant. Again $X_{\mathrm{I}}=0$. Hence $\theta=90^{\circ}$ or $270^{\circ}$. The latter gives the positive semidefinite symmetric magnification

$\mathbf{X}_{\mathrm{sym}}=\left(\begin{array}{ll}2 & 1 \\ 1 & 2\end{array}\right)$

or, equivalently, $1\{135\} 3\{45\}$. Thus generalized magnification $\left(\begin{array}{cc}1 & 2 \\ -2 & -1\end{array}\right)$ is equivalent to symmetric magnification $1\{135\} 3\{45\}$ followed by clockwise rotation through $90^{\circ}$. The magnification is illustrated in Figure 6.

Figure 9 illustrates generalized magnification by $\left(\begin{array}{ll}1 & 5 \\ 1 & 3\end{array}\right)$. The negative determinant implies reflection. Equation 36 gives $\tan 2 \theta=3 /(-1)$. Thus $2 \theta=108.4^{\circ}$ or $288.4^{\circ}$ and $\theta=54.2^{\circ}$ or $144.2^{\circ}$. The corresponding symmetric magnifications are

$$
\mathbf{X}_{\mathrm{sym}}=\left(\begin{array}{cc}
-0.3162 & 0.9487 \\
0.9487 & 0.3162
\end{array}\right)\left(\begin{array}{ll}
1 & 5 \\
1 & 3
\end{array}\right)=\left(\begin{array}{ll}
0.6325 & 1.2649 \\
1.2649 & 5.6921
\end{array}\right)
$$

and its negative respectively. The former is equivalent to $0.3\{166.7\} 5.9\{76.7\}$ and is positive semidefinite. Hence magnification $\left(\begin{array}{ll}1 & 5 \\ 1 & 3\end{array}\right)$ is equivalent to symmetric magnification $0.3\{166.7\} 5.9\{76.7\}$ followed by reflection in the meridian at angle $54.2^{\circ}$.

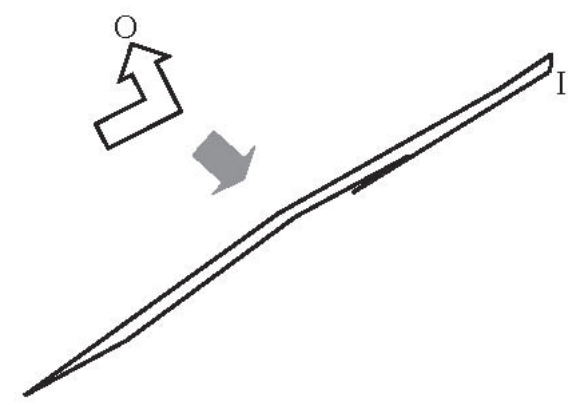

Figure 9 The image I of an object $\mathrm{O}$ under asymmetric magnification $\left(\begin{array}{ll}1 & 5 \\ 1 & 3\end{array}\right)$. The magnification is equivalent to symmetric magnification $0.3\{166.7\} 5.9\{76.7\}$ followed by reflection in the meridian at angle $54.2^{\circ}$. 


\section{Concluding remarks}

Generalized magnification in linear optics is very much more complicated than in Gaussian optics. Instead of being a scalar it is a $2 \times 2$ matrix. Magnifications can take the form of familiar magnifications, distortions, inversions, rotations, reflections and combinations of these. We have illustrated generalized magnification in a number of representative cases.

Magnifications that are positive semidefinite (neither principal magnification is negative) are relatively easy to interpret. The principal meridians of the magnification are orthogonal; the object is magnified along the principal meridians by the corresponding principal magnifications. One can construct magnification crosses just as one does in the case of power crosses.

Generalized magnifications can take the form of rotations and reflections. These are also easy to interpret.

Every other generalized magnification can be interpreted as a positive semidefinite magnification followed by either a rotation or a reflection. How one does so has been described above.

The interpretation of generalized magnification offered here does not exclude other possible interpretations. One could instead choose to have the rotation or reflection first with the symmetric magnification second. Angles and matrices would then differ in general. And there are other possibilities. The decomposition described here, however, seems the best, or equal to the best, for optometric applications in that it most closely resembles concepts already familiar in optometry including the power cross, principal powers and principal meridians.

One last point is, perhaps, worth making. Generalized magnification changes the appearance of an object; but ${ }^{\circ}$ no always. If the magnification happens to be a symmetry of the object then the image will look as if there has been no change. Rotation through 90 about the centre will change the appearance of an equilateral triangle but not of a square.

In Part 2 we shall generalize magnification still further to a magnification that changes not only the appearance but also the position of an object ${ }^{26}$.

\section{Acknowledgements}

I gratefully acknowledge support from the National Research Foundation. I thank J R Cardoso and S D Mathebula for comments on the manuscript.

\section{References}

1. Freeman MH. Optics. 10th ed. Butterworth-Heinemann, Oxford, 199093.

2. Das P. Lasers and Optical Engineering. Springer, New York, 1991, 18.

3. Smith WJ. Modern Optical Engineering. 4th ed. McGrawHill, New York, 200826.

4. Meyer-Arendt JR. Introduction to Classical and Modern Optics. 3rd ed. Prentice-Hall, Englewood Cliffs, NJ, 1989 41.

5. Keating MP. Geometric, Physical, and Visual Optics. 2nd Ed. Butterworth-Heinemann, Boston, 200256.

6. Rabbetts RR. Bennett \& Rabbetts' Clinical Visual Optics. 4th ed. Butterworth-Heinemann, Edinburgh, 200710.

7. Woo GC, Mah-Leung A. The term magnification. Clin Exper Optom 200184 113-119.

8. Keating MP. A matrix formulation of spectacle magnification. Ophthal Physiol Opt 19822 145-158.

9. Harris WF. Stigmatic optical systems. Optom Vis Sci 2004 81 947-952.

10. Harris WF. Proper and improper stigmatic optical systems. Optom Vis Sci 200481 953-959.

11. Harris WF Magnification, blur, and ray state at the retina for the general eye with and without a general optical instrument in front of it. 1. Distant objects. Optom Vis Sci 2001 78 888-900.

12. Harris WF Magnification, blur, and ray state at the retina for the general eye with and without a general optical instrument in front of it. 2. Near objects. Optom Vis Sci 200178 901-905.

13. Harris WF. Image size magnification and power and dilation factors for optical instruments in general. Ophthal Physiol Opt 200323 251-261.

14. Harris WF. Near image size magnification for optical instruments in general. Optom Vis Sci 200380 606-608.

15. Harris WF. Special rays and structures in general optical systems. S Afr Optom 201069 51-57.

16. Lütkepohl H. Handbook of Matrices. Wiley, Chichester, 1996 48, 63, 64, 254.

17. Horn RA, Johnson CR. Matrix Analysis. Cambridge Univ Press, Cambridge, 1985 5, 13, 35, 57, 396.

18. Zwillinger D. CRC Standard Mathematical Tables and Formulae. 31st ed. Chapman \& Hall/CRC, Boca Raton, 2003 84, 152-153.

20. Harris WF. Nodes and nodal points and lines in eyes and other optical systems. Ophthal Physiol Opt 201030 24-42

21. Harris WF. Quantitative analysis of transformed transferences of optical systems in a space of augmented Hamiltonian matrices. SAfr Optom 200766 62-67. 
22. Harris WF. Interconverting the matrix and principal meridional representations of dioptric power in general including powers with nonorthogonal and complex principal meridians. Ophthal Physiol Opt 200121 247-252.

23. Grossman SI. Elementary Linear Algebra. 3rd ed. Wadsworth, Belmont CA, 1987 263, 320, 335.

24. Anton H, Rorres C. Elementary Linear Algebra with Applications. Wiley, New York, 1987 267, 280.

25. Bernstein DS. Matrix Mathematics: Theory, Facts, and Formulas. 2nd ed. Princeton University Press, Princeton, $2009179,393$.

26. Harris WF. Generalized magnification in visual optics. Part 2: Magnification as affine transformation. S Afr Optom 2010 69 in press. 